Math. Model. Nat. Phenom.

Vol. 6, No. 1, 2011, pp. 48-61

DOI: $10.1051 / \mathrm{mmnp} / 20116103$

\title{
Solitary Structures Sustained by Marangoni Flow
}

\author{
L.M. Pismen ${ }^{1}$ \\ Department of Chemical Engineering and Minerva Center for \\ Nonlinear Physics of Complex Systems, Technion, 32000 Haifa, Israel
}

\begin{abstract}
We construct interfacial solitary structures (spots) generated by a bistable chemical reaction or a non-equilibrium phase transition in a surfactant film. The structures are stabilized by Marangoni flow that prevents the spread of a state with a higher surface tension when it is dynamically favorable. In a system without surfactant mass conservation, a unique radius of a solitary spot exists within a certain range of values of the Marangoni number and of the deviation of chemical potential from the Maxvell construction, but multiple spots attract and coalesce. In a conservative system, there is a range of stable spot sizes, but solitary spots may exist only in a limited parametric range, beyond which multiple spots nucleate. Repeated coalescence and nucleation leads to chaotic dynamics of spots observed computationally in Ref. [1].
\end{abstract}

Key words: solitary structures, Marangoni convection, coalescence, nucleation AMS subject classification: 76D45, 76V05

\section{Introduction}

This paper, presented at the Haifa workshop in June 2009 and dedicated to the memory of Sasha Golovin, is a sequel to our joint work [1], which we had planned to continue before Sasha's lethal disease was diagnosed.

The main motivation of this study is a quest for dynamic non-equilibrium structures resisting the coarsening tendency common to system relaxing to equilibrium. This tendency is overcome in pattern-forming non-equilibrium systems, which are most easily constructed

${ }^{1}$ Email: pismen@technion.ac.il 
by combining a short-scale "activator" and a long-range "inhibitor". Both roles are played by diffusive chemical species in classical Turing patterns [2] - see [3, 4] for extensive review - but the role of an "inhibitor", which arrests spreading of one of alternative states of an autocatalytic chemical reaction, can be also played by a physical process coupled to changes of chemical composition.

The influence of Marangoni flow on chemical waves in a bistable system has been first investigated theoretically for shallow layers enabling lubrication approximation $[5,6]$. The chemical model was later extended to the two-component FitzHugh-Nagumo system, which is pattern-forming in its own right [7]. Further numerical modeling confirmed that Marangoni flow may change relative stability of the two stable steady states, reversing in some cases the direction of propagation of the front [8].

A possibility of formation of interfacial solitary structures generated by chemical instabilities and stabilized by Marangoni flow has been demonstrated earlier by the author [9]. In this communication we will elaborate the results of this work and extend them to a conservative system studied numerically in Ref. [1] to give a theoretical explanation to the phenomenon of chaotic spot dynamics observed there.

\section{Basic equations}

Consider an insoluble surfactant taking part in an autocatalytic chemical reaction. The surface concentration $\theta$ obeys the convective reaction-diffusion equation

$$
\theta_{t}=D \nabla^{2} \theta-\nabla \cdot(\mathbf{v} \theta)+\tau^{-1}[f(\theta)-\mu],
$$

where $D$ is the surface diffusivity, $\nabla$ is the two-dimensional vector differential operator, $\tau$ is the characteristic relaxation time. The algebraic part $f(\theta)-\mu$ may represent the dimensionless net surfactant source due to chemical reactions and exchange with the gas phase. Alternatively, the same model can be motivated thermodynamically. In this formulation, the function $f(\theta)$ would be the derivative of the Helmholtz free energy with respect to the concentration, and $\mu$, the chemical potential. If the function $f(\theta)$ is not monotonic, the surfactant undergoes within a certain range of $\mu$ spinodal decomposition leading to separation into two surface phases with different concentrations. A purely thermodynamic setting is not suitable for our study, since, in a closed system, the surface phase with a lower surface tension will always tend to spread; this spreading will be enhanced by Marangoni flow, and no non-equilibrium structures may form. The system can be sustained, however, far from equilibrium by external fluxes. If, for example, the surface is illuminated, as suggested in Ref. [1], and the light absorption coefficient is concentration-dependent, the thermodynamically disadvantageous but stronger-adsorbing phase may lower its surface tension due to the thermal effect and start spreading by the action of the Marangoni flow.

We are interested in the case when at least two stable stationary solutions exist; therefore the function $f(\theta)-\mu$ should have three zeroes, and its derivative at the smallest and largest zero should be negative. The simplest function possessing this property, which is commonly 


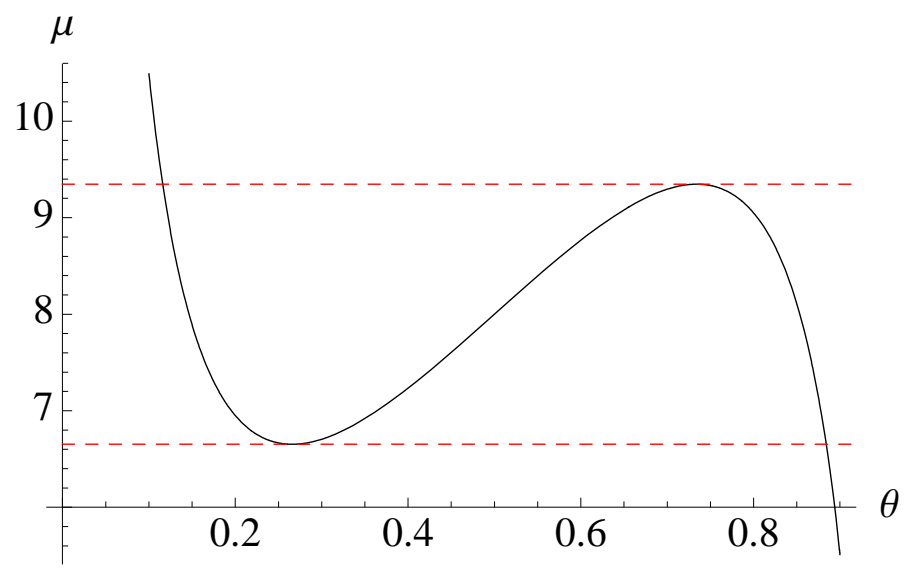

Figure 1: The model function $f(\theta)$ with $\nu=16$; the dashed lines delimit the bistability interval.

used in theoretical studies, is a cubic. We will use here, however, a more realistic model function, which (similar to the van der Waals interaction function) ensures that the algebraic part of Eq. (2.arabicequation) always has zeroes lying within the interval $0<\theta<1$ :

$$
f(\theta)=\frac{1}{\theta}-\frac{1}{1-\theta}+\nu \theta
$$

The system is bistable within a finite range of $\mu_{c}^{-}<\mu<\mu_{c}^{+}$at $\nu>8$ (Fig. 1).

Taking $\tau$ as the time scale and scaling the coordinates by the diffusional length $\sqrt{D / \tau}$ (which determines the thickness of a front separating the two alternative homogeneous steady states) and the velocity, by $\sqrt{D \tau}$, we rewrite Eq. (2.arabicequation) in the dimensionless form

$$
\theta_{t}=\nabla^{2} \theta-\nabla \cdot(\mathbf{v} \theta)+f(\theta)+\mu
$$

(2.arabicequation)

In this model, studied by the author earlier using a different model function [9], the surfactant is not conserved, as it is assumed that it may be produced and consumed as a result of chemical reactions. An alternative model of a Cahn-Hilliard type that conserves the "order parameter" (surfactant concentration) [1] has the form

$$
\begin{array}{ll}
\theta_{t}+\nabla \cdot(\mathbf{v} \theta)+\nabla \cdot(\Gamma(\theta) \nabla \mu)=0, & \text { (2.arabicequation) } \\
\nabla^{2} \theta+f(\theta)-\mu=0 . & \text { (2.arabicequation) }
\end{array}
$$

In this model, $\mu$ is not a constant as in Eqs. (2.arabicequation) but an additional variable the dimensionless chemical potential; the characteristic interface thickness in this model is the healing length $\sqrt{K / \tau}$ depending on the rigidity coefficient $K$, which determines the line tension of the boundary between dense and dilute surfactant phases. This coefficient has to replace $D$ in the above definitions of the length and velocity scales. $\Gamma$ is the dimensionless mobility related near a homogeneous state $\theta=\theta^{ \pm}$to the diffusivity by $D=-K \Gamma f^{\prime}\left(\theta^{ \pm}\right)$. 


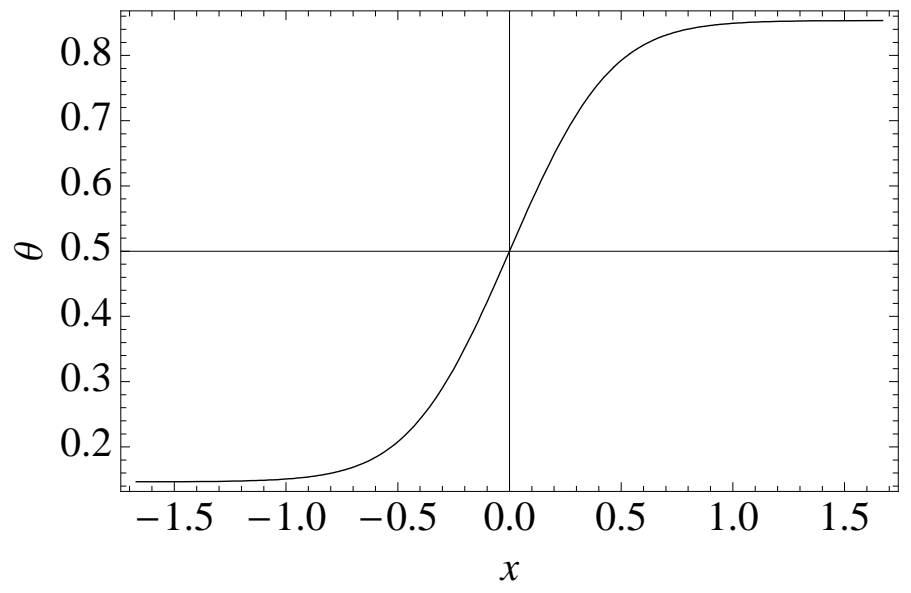

Figure 2: A typical stationary front solution $\left(\nu=16, \mu_{0}=8\right)$.

Equation (2.arabicequation) or (2.arabicequation) has a stationary front solution $\theta=$ $\theta_{0}(x)$ at a Maxwell point $\mu=\mu_{0}$ lying within the bistability interval; e.g. for $\nu=16$ we have $\mu_{0}=8$, and the alternative stationary states approached at $x \rightarrow \pm \infty$ are $\theta^{ \pm}=\frac{1}{4}(2 \pm \sqrt{2})$ (Fig. 2). The solution is also valid in a moving frame when the front is advected with a constant velocity, but is distorted when velocity changes along the $x$ axis. The front is set into motion when $\mu \neq \mu_{0}$ or when it is curved.

\section{Marangoni flow}

\subsection{Hydrodynamic model}

The interfacial flow velocity appearing in Eqs. (2.arabicequation), (2.arabicequation) is induced by the Marangoni force due to an instantaneous distribution of surface tension $\sigma(\mathbf{x})$ that corresponds to a given instantaneous surface concetration profile. Further on, we assume a linear dependence $\sigma=\sigma_{0}-\sigma_{1} \theta$. Under usual conditions, inertial terms can be neglected, and the Stokes equation is applicable. Furthermore, there are no sources of vertical vorticity in this configuration, and the solution can be expressed though a single flow potential $\chi$ obeying the biharmonic equation

$$
\left(\nabla^{2}+\partial_{z}^{2}\right)^{2} \chi=0
$$

(3.arabicequation)

where we separated the derivative with respect to the vertical coordinate $\partial_{z}$. For our purpose, we need only to compute the two-dimensional horizontal velocity vector $\mathbf{v}=\nabla \chi_{z}(0)$ by solving Eq. (3.arabicequation) in an infinitely deep and infinitely extended fluid volume $z<0$ with the Marangoni boundary conditions on the undeformable free boundary $z=0$. The dimensionless form of the boundary condition is

$$
\chi(\mathbf{x}, 0)=0, \quad \chi_{z z}(\mathbf{x}, 0)=M \nabla \theta
$$

(3.arabicequation) 
where $M$ is the Marangoni number based on either diffusional or healing length, respectively, in the non-conservative and conservative models:

$$
M=\frac{\sigma_{1}}{\eta} \sqrt{\frac{\tau}{D}} \quad \text { or } \quad M=\frac{\sigma_{1}}{\eta} \sqrt{\frac{\tau}{K}},
$$

$\eta$ being the dynamic viscosity. The solution is expressed [9] as an integral over the interfacial plane $z=0$ :

$$
\mathbf{v}(\mathbf{x})=-\frac{M}{4 \pi} \int \frac{\nabla \theta(\mathbf{x}+\mathbf{r})}{|\mathbf{r}|} d^{2} \mathbf{r} .
$$

This solution exists, provided $\theta(\mathbf{x})=$ const at $|\mathbf{x}| \rightarrow \infty$. Using Eq. (3.arabicequation) in Eq. (2.arabicequation) or (2.arabicequation) yields an integro-differential equation of the surface concentration. This equation is nonlocal so that the motion is dependent on the instantaneous distribution of domains occupied by the alternative states in the entire region.

\subsection{Flow far for the front}

On distances far exceeding the front thickness, which is unity in our dimensionless formulation, the concentration distribution can be considered as stepwise. Using the discontinuous function $\theta(\mathbf{x})$ that approaches (at $\left|\mu-\mu_{0}\right| \ll 1$ ) the two alternative values $\theta=\theta^{ \pm}$in the domains separated by a sharp front contour $\mathcal{C}$ brings Eq. (3.arabicequation) to the form

$$
\mathbf{v}(\mathbf{x})=-\frac{M \Delta}{4 \pi} \oint_{\mathcal{C}} \frac{\mathbf{n}}{r(s)} d s,
$$

where $\Delta=\theta^{+}-\theta^{-}$; the contour is parametrized by the arc length $s$, and $r(s)=|\mathbf{x}-\mathbf{y}(s)|$ is the distance from the reference point to a point on the contour; by convention, the normal $\mathbf{n}$ is directed towards the domain occupied by the upper state.

This formula can be used to compute the flow induced by a circular spot of the radius $R \gg 1$ occupied by the lower state on the infinite interfacial plane occupied by the upper state:

$$
\begin{aligned}
v(\rho) & =-\frac{M \Delta}{2 \pi} \int_{0}^{\pi} \frac{\cos \alpha d \alpha}{\sqrt{1+(\rho / R)^{2}-2(\rho / R) \cos \alpha}} \\
& =-\frac{M \Delta}{2 \pi}\left[\frac{R^{2}+\rho^{2}}{\rho(R+\rho)} \mathbf{K}\left(\frac{4 R \rho}{R^{2}+\rho^{2}}\right)-\frac{R+\rho}{\rho} \mathbf{E}\left(\frac{4 R \rho}{R^{2}+\rho^{2}} \cdot\right. \text {. Ara,bicequation) }\right.
\end{aligned}
$$

where $\rho, \alpha$ are polar coordinates, and $\mathbf{K}, \mathbf{E}$ are complete elliptic integrals. The radial flow velocity vanishes at $\rho=0$ and decays as

$$
v \asymp-\frac{M \Delta}{4}\left(\frac{R}{\rho}\right)^{2} \quad \text { at } \quad \rho \rightarrow \infty,
$$




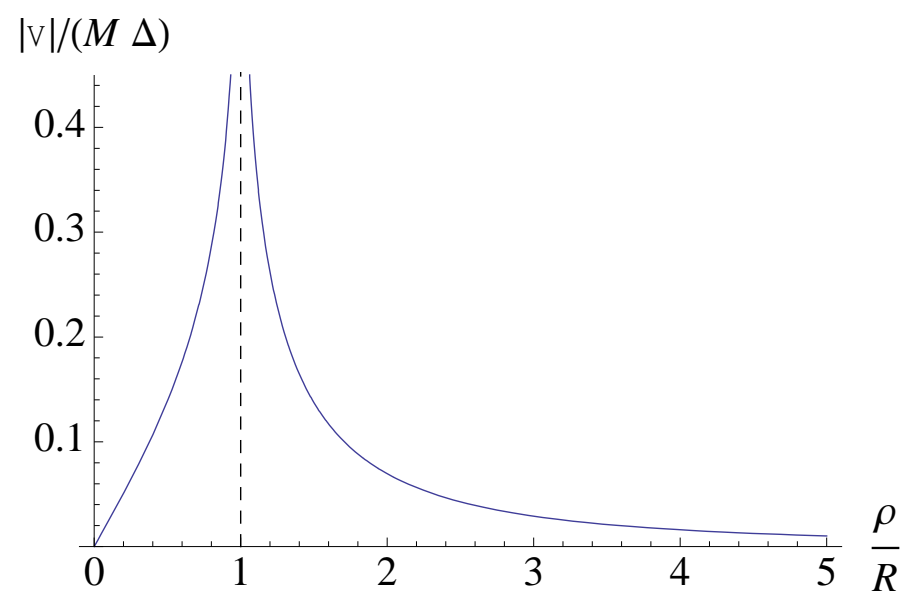

Figure 3: The interfacial radial velocity in and around a solitary spot.

The solution drawn in Fig. 3 diverges in the vicinity of the front:

$$
v \asymp-\frac{M \Delta}{2 \pi} \ln \left|\frac{8 R}{e^{2}(\rho-R)}\right| \quad \text { at } \quad|\rho-R| \ll R \text {. }
$$

(3.arabicequation)

This divergence does not allow us to compute the flow velocity where we need it most at the front itself, and therefore the question of the influence of Marangoni flow on the motion of the front cannot be answered before the singularity is resolved by the asymptotic procedure to be described below. The far field solution (3.arabicequation) or its asymptotics (3.arabicequation) can be applied, however, to describe interaction between spots removed at distances large compared to there radii (where their circular shape is only weakly distorted).

Separate spots are attracted one to the other by the induced inward flow. If there is a number of spots removed at distances far exceeding the diffusional length, the induced flow is additive, since the Stokes problem is linear. At any fixed distance, a larger velocity is induced by larger spot, and the flow is always centripetal, so that spots originally separated by a distance $L$ eventually coalesce. The coalescence time for a pair of spots with the radii $R_{1}, R_{2}$ can be computed, neglecting a fast finite stage when the asymptotics (3.arabicequation) is no longer applicable and shape distortion is substantial, by solving the equation for the distance between the centers of two spots $\ell(t)$

$$
\frac{d \ell}{d t}=-\frac{M \Delta}{4}\left[\left(\frac{R_{1}}{\ell}\right)^{2}+\left(\frac{R_{1}}{\ell}\right)^{2}\right]
$$

Integrating this gives the coalescence time

$$
T=-\frac{4}{3 M \Delta} \frac{L^{3}}{R_{1}^{2}+R_{2}^{2}}
$$

It has to be emphasized that long-distance attraction of spots is a nonlocal effect dependent on the depth of the liquid layer. The interaction length is of the same order of magnitude as the depth of the liquid layer, and in shallow water the effect disappears altogether. 


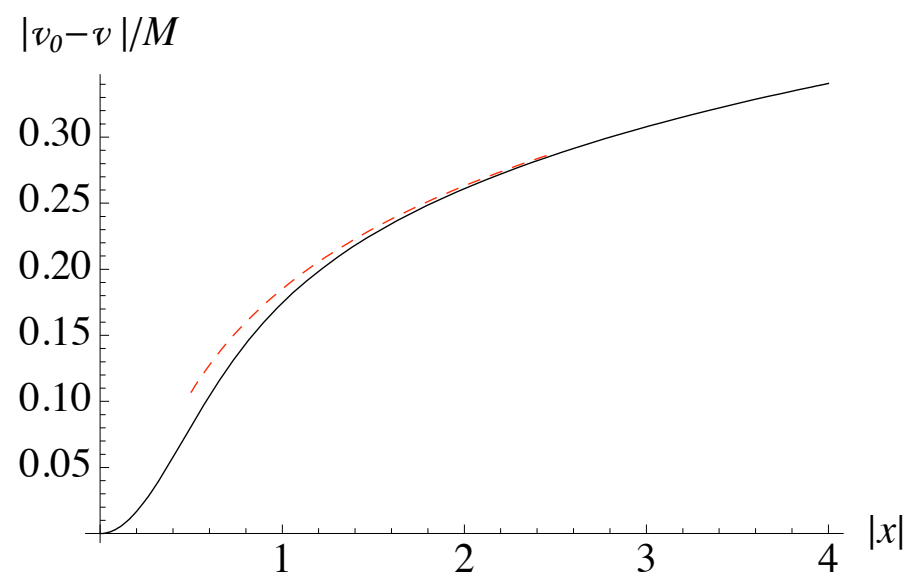

Figure 4: The velocity profile in the vicinity of the front (the maximum velocity $v_{0}$ at $x=0$ is taken as zero level) The dashed line denotes the asymptotics $\beta(x) \asymp|x|$.

\subsection{Flow in the vicinity of the front}

The flow velocity in the front region is coupled to the concentration profile. It is computed with the help of a two-tier asymptotic procedure [10,9]. First, we remove from the integral (3.arabicequation) a short arc segment $|\alpha|<\delta \ll 1$ responsible for the divergence. On the outer segment of the circular contour we compute:

$$
v^{(o)}(R)=-\frac{M \Delta}{2 \pi} \lim _{\delta \rightarrow 0} \int_{\delta}^{\pi} \frac{\cos \alpha d \alpha}{\sin (\alpha / 2)}=-\frac{M \Delta}{2 \pi} \ln \frac{4}{e^{2} \delta} .
$$

On the removed segment, the finite front thickness has to be taken into account by replacing the step function that has lead us to Eq. (3.arabicequation) by a smooth concentration profile. The stationary profile $\theta=\theta_{0}(x)$ can be used here, assuming that it is only weakly distorted by the flow. Equation (3.arabicequation) is rewritten then on the segment $|\alpha|<\delta$ as

$$
v^{(i)}(x)=-\frac{M}{2 \pi} \int_{-\infty}^{\infty} \theta_{0}^{\prime}(\xi) d \xi \int_{0}^{\delta} \frac{R d \alpha}{\sqrt{\left(x-\xi^{2}\right)+R^{2} \alpha^{2}}} \asymp-\frac{M}{2 \pi} \int_{-\infty}^{\infty} \theta_{0}^{\prime}(\xi) \ln \frac{2 R \delta}{|x-\xi|} d \xi .
$$

(3.arabicequation)

The last expression is valid in the limit $R \delta \gg 1$, i.e. when the length of the inner contour is much larger than the front thickness.

The auxilliary value $\delta$ falls out when both integrals (3.arabicequation) and (3.arabicequation) are added up. The resulting finite expression for the flow velocity in the front region is

$$
v(x)=-\frac{M \Delta}{2 \pi} \ln \frac{8 R}{e^{2} \beta(x)} ; \quad \ln \beta(x)=\frac{1}{\Delta} \int_{-\infty}^{\infty} \theta_{0}^{\prime}(\xi) \ln |x-\xi| d \xi . \quad \text { (3.arabicequation) }
$$

Since $\theta_{0}^{\prime}(\xi) \rightarrow 0$ at $|\xi| \rightarrow \infty, \beta(x) \asymp|x|$ at $|x| \gg 1$. Thus, the outer asymptotics of the inner solution (3.arabicequation) matches the inner asymptotics (3.arabicequation) of the outer 
solution (3.arabicequation) with $\rho=R+x$. The function $\beta(x)$ can be computed numerically using the basic front solution $\theta_{0}(x)$. The resulting velocity profile in the front region for $\nu=16$ is plotted in Fig. 4. The uniformly valid composite expansion can be obtained by adding up Eqs. (3.arabicequation), (3.arabicequation) and extracting their common limit (3.arabicequation).

\section{Non-conservative system}

With the flow velocity in the front region known, the front propagation speed can be computed. Consider first the non-conservative system. In Ref. [9] only an estimate based on the maximum flow velocity has been given, but the exact result can be obtained semi-analytically by considering the actual concentration profile to be a perturbation of the basic front solution $\theta_{0}(x)$ and computing the solvability condition of the perturbation expansion [4].

We consider the physically relevant distinguished limit when the curvature of the front $\kappa=R^{-1}$, the front propagation speed $c$, the flow velocity $v$, and the deviation from the Maxwell construction $\left|\mu-\mu_{0}\right|$ are all small and of the same order of magnitude. This can be formalized by introducing a book-keeping small parameter $\epsilon$ and replacing $\kappa \rightarrow$ $\epsilon \kappa, c \rightarrow \epsilon c, v \rightarrow \epsilon v, \mu \rightarrow \mu_{0}+\epsilon \mu_{1}$. In accordance to a common procedure of the theory of front dynamics [4], a propagating front is constructed as a stationary solution of Eq. (2.arabicequation) in a local coordinate frame moving with the front speed, as yet undetermined. Up to the first order in $\epsilon$, this equation reads

$$
\theta_{x x}+f(\theta)-\mu_{0}+\epsilon \Psi[\theta(x)]=0, \quad \Psi[\theta]=-\mu_{1}+(c+\kappa) \theta_{x}-(v \theta)_{x} . \quad \text { (4.arabicequation) }
$$

Expanding $\theta=\theta_{0}+\epsilon \theta_{1}+\ldots$ yields in the first order

$$
\theta_{1}^{\prime \prime}(x)+f^{\prime}\left(\theta_{0}\right) \theta_{1}+\Psi\left[\theta_{0}(x)\right]=0 .
$$

(4.arabicequation)

The homogeneous part of this equation has a zero eigenvalue corresponding to the translational symmetry of the zero-order equation. The solvability condition of the inhomogeneous equation requires $\Psi\left[\theta_{0}\right]$ to be orthogonal to the corresponding eigenfunction $\theta_{0}^{\prime}(x)$ :

$$
\begin{aligned}
& 0= \int_{-\infty}^{\infty} \theta_{0}^{\prime}(x) \Psi\left[\theta_{0}\right] d x=-\mu_{1} \Delta+(c+\kappa) K+\frac{M}{\pi} I, \quad \text { (4.arabicequation) } \\
& K=\int_{-\infty}^{\infty}\left[\theta_{0}^{\prime}(x)\right]^{2} d x=\int_{\theta^{-}}^{\theta^{+}} \sqrt{2 Y(\theta)} d \theta, \quad \text { (4.arabicequation) } \\
& Y(\theta)=\int_{\theta^{-}}^{\theta} f(\vartheta) d \vartheta-\mu_{0}\left(\vartheta-\theta^{-}\right), \\
& I=\int_{-\infty}^{\infty} \theta_{0}^{\prime}(x) \frac{d}{d x}\left[\theta_{0}(x) \ln \frac{8 R}{e^{2} \beta(x)}\right] d x=K \ln \frac{8 R}{e^{2}}+J(\text { arabicequation) } \\
& J=\frac{1}{\Delta} \int_{-\infty}^{\infty} \theta_{0}^{\prime \prime}(x) \theta_{0}(x) d x \int_{-\infty}^{\infty} \theta_{0}^{\prime}(\xi) \ln |x-\xi| d \xi
\end{aligned}
$$




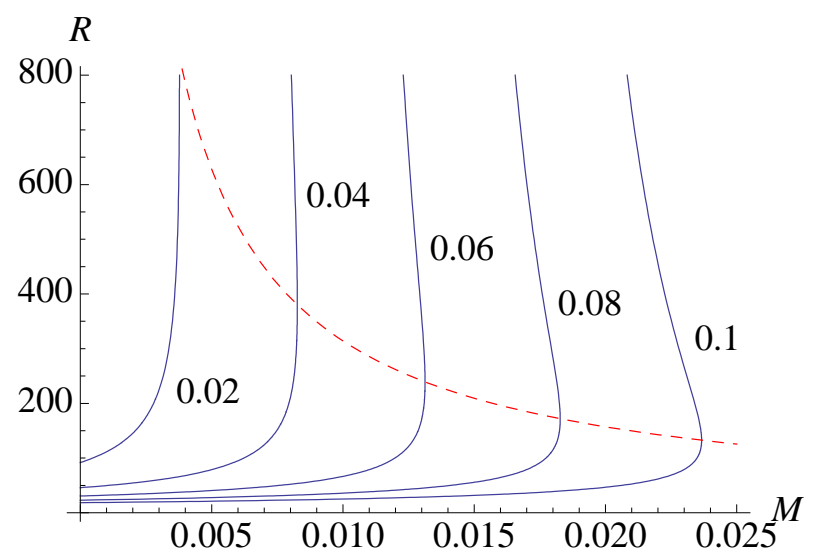

$\mathrm{b}$

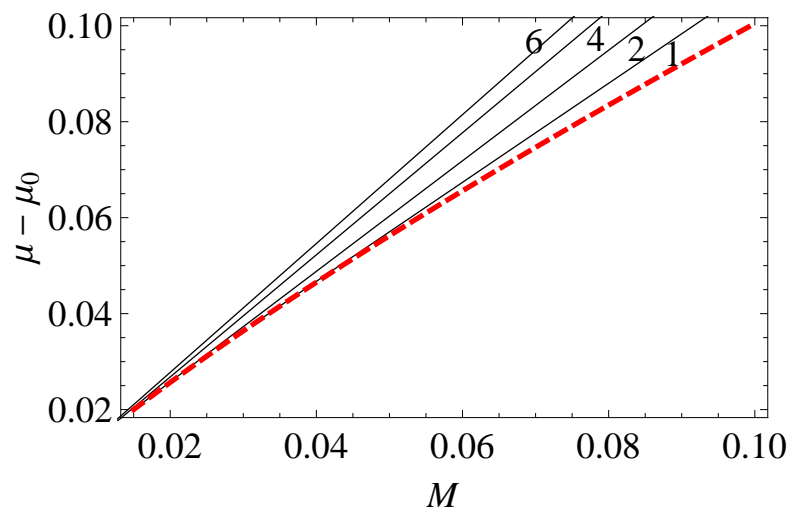

Figure 5: (a) The dependence $R_{s}(M)$ at different values of $\mu-\mu_{0}$ (shown at the respective curves). Stable solutions lie above dashed line. (b) Loci of the $R_{s}=$ const in the parametric plane $M, \mu$. The numbers at the curves indicate the values of $10^{-2} R_{s}$.

$$
=\frac{1}{\Delta} \int_{\theta^{-}}^{\theta^{+}} \frac{\theta[\mu-f(\theta)]}{\sqrt{2 Y(\theta)}} d \theta \int_{\theta^{-}}^{\theta^{+}} \ln |x(\theta)-x(\vartheta)| d \vartheta \text { (4.arabicequation) }
$$

The integral $I$ has been rearranged here using Eq. (3.arabicequation) and integrating by parts and expressed through the inverse of $\theta_{0}(x), x(\theta)=\int[2 Y(\theta)]^{-1 / 2} d \theta$ to facilitate further computation. The integrals $K, J$, dependent only on the parameter $\nu$, are evaluated numerically. Collecting the results yields the expression for the front propagation velocity (eikonal equation)

$$
c=\frac{\Delta}{K}\left(\mu-\mu_{0}\right)-\frac{1}{R}-\frac{M}{\pi} \ln \frac{R}{R_{0}}, \quad R_{0}=\frac{1}{8} \exp \left(2-\frac{J}{K \Delta}\right) . \quad \text { (4.arabicequation) }
$$

This expression reaches a maximum at $R=R_{c}=\pi / M$, and no solutions exist when $M$ exceeds the critical value defined implicitly by the relation

$$
\frac{\pi \Delta}{K}\left(\mu-\mu_{0}\right)=M\left(1+\ln \frac{\pi}{M R_{0}}\right) .
$$

Stable stationary spots with a radius $R>R_{c}$ defined implicitly by the relation $c=0$ exist at sufficiently large values of $\mu-\mu_{0}$ (making the lower state dynamically advantageous). As an example, the dependence $R_{s}(M)$ at different values of $\mu-\mu_{0}$ and a contour map of $R_{s}$ in this parametric plane are shown in Fig. 5 for $\nu=16$. The values of the integrals for this case are $K \approx 0.385, J \approx 0.345, R_{0} \approx 0.26$.

Thus, Marangoni flow serves as an efficient mechanism preventing the spread of the state with a higher surface tension when it is dynamically favorable. Stationary spots with a finite radius, at which the inward flow is compensated by the chemical propagation speed, exist 
above the dashed curve in Fig. 5. This, however, still does not prevent coarsening in an ensemble of spots, which may be formed at far removed locations. Attracting spots coalesce in a finite time, as per Eq. (3.arabicequation); following this, the combined spot relaxes to a circular shape with the stationary radius defined by Eq. (4.arabicequation). Multiple spots can survive only in the presence of global interactions mediated by the conservation law, as will be argued below.

\section{Conservative system}

\subsection{Inner solution}

The conservative system (2.arabicequation), (2.arabicequation) is expanded in the same way as in the preceding Section $[4,11]$. Assuming $\Gamma=$ const, Eq. (2.arabicequation) is rewritten in the comoving frame to the first order in $\epsilon$ as

$$
\frac{d}{d x}\left[\Gamma \mu_{1}^{\prime}(x)+(v-c) \theta_{0}(x)\right]=0 .
$$

(5.arabicequation)

The front velocity should match the surfactant fluxes $j^{ \pm}$on both sides of the front, and is determined by the material balance relation obtained by integrating Eq. (5.arabicequation) over an interval $|x|<\lambda$, which is sufficiently large for the alternative states with $\theta_{0}= \pm 1$ to be approached at its ends:

$$
c=\left(j^{+}-j^{-}\right) / \Delta, \quad j^{ \pm}=\Gamma \mu^{\prime}( \pm \lambda)+v( \pm \lambda) \theta^{ \pm} .
$$

(5.arabicequation)

Integrating Eq. (5.arabicequation) twice yields

$$
\mu_{1}(x)=\bar{\mu}_{1}+\Gamma^{-1}\left[j x+\int_{0}^{x}(c-v) \theta_{0}(x) d x\right]
$$

(5.arabicequation)

where $\bar{\mu}_{1}=\mu_{1}(0)$ and $j$ are integration constants; the latter equals to the flux through the interface $\left(j^{+}+j^{-}\right) / \Delta$ determined by matching with the far field solution.

The first-order expansion of Eq. (2.arabicequation) has the form (4.arabicequation) with the inhomogeneity $\Psi[\theta]=-\mu_{1}+\kappa \theta_{x}$. Its solvability condition is computed using Eq. (5.arabicequation) in the same way as in Eq. (4.arabicequation):

$$
\begin{aligned}
0 & =-\bar{\mu}_{1} \Delta+K \kappa+\frac{\mathcal{K}}{\Gamma} c+\frac{\mathcal{I}}{\Gamma} \frac{M}{\pi} \\
\mathcal{K} & =\int_{-\infty}^{\infty} \theta_{0}^{\prime}(x) d x \int_{0}^{x} \theta_{0}(\xi) d \xi=\int_{\theta^{-}}^{\theta^{+}} d \theta \int_{\theta^{0}}^{\theta} \frac{\vartheta}{\sqrt{2 Y(\vartheta)}} d(\text { 5.arabicequation) } \\
\mathcal{I} & =\int_{-\infty}^{\infty} \theta_{0}^{\prime}(x) \int_{0}^{x} \theta_{0}(\xi) \ln \frac{8 R}{e^{2} \beta(\xi)} \xi=\mathcal{K} \ln \frac{8 R}{e^{2}}-\mathcal{J}, \quad \text { (5.arabicequation) } \\
\mathcal{J} & =\int_{-\infty}^{\infty} \theta_{0}^{\prime}(x) d x \int_{0}^{x} \theta_{0}(\xi) d \xi \int_{-\infty}^{\infty} \theta_{0}^{\prime}(\zeta) \ln |\xi-\zeta| d \zeta \\
& =\int_{\theta^{-}}^{\theta^{+}} d \theta \int_{\theta^{0}}^{\theta} \frac{\vartheta}{\sqrt{2 Y(\vartheta)}} d \vartheta \int_{\theta^{-}}^{\theta^{+}} \ln |x(\vartheta)-x(\zeta)| d \zeta . \quad \text { (5.arabicequation) }
\end{aligned}
$$




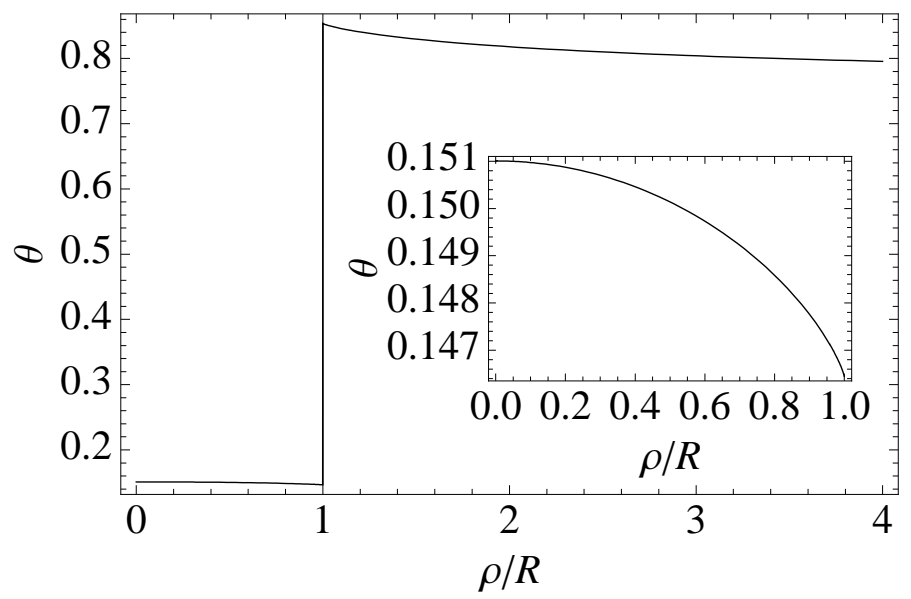

Figure 6: The extremal far field concentration profile in and around the largest solitary spot obtained by solving Eq. (5.arabicequation) with $M R_{c} \approx 7.305$. Inset: the concentration profile within the spot redrawn at a larger scale

The origin of the $x$ axis is chosen here to coincide with the level $\theta^{0}$ corresponding to the intermediate unstable stationary state. The term proportional to $j$ vanishes identically since $\theta_{0}(x)$ is even. The solvability condition relates the representative value $\bar{\mu}_{1}$ of the chemical potential at the front with the external fluxes dependent on the gradient of the chemical potential and flow velocity on both sides of the front.

The two relations (5.arabicequation) and (5.arabicequation) are still not sufficient to determine the front propagation speed: since the material balance operates globally, far field equations have to be solved to compute the flux at the front location. On the other hand, since the chemical potential at the front should be close to the Maxwell construction, its shift given by Eq. (5.arabicequation) only weakly affects the concentrations in the far field, and can be neglected when the latter are computed.

\subsection{Outer solution}

In the far field, the rigidity term in Eq. (2.arabicequation) is negligible, so that the quasiequilibrium relation $\mu=f(\theta)$ holds, but the concentration does not need to be close to $\theta^{ \pm}$. The concentration profile within and around a solitary spot can be obtained by solving Eq. (2.arabicequation) in polar coordinates, with $\mu$ given by the above quasi-equilibrium relation. When the spot is stationary, the flux should vanish, and therefore the stationary equation can be integrated once to yield

$$
f^{\prime}(\theta) \theta^{\prime}(\rho)=v(\rho) \theta
$$

(5.arabicequation)

where $v(\rho)$ is given by Eq. (3.arabicequation). The boundary condition at the circular front $\rho=R$ is $\theta(R)=\theta^{+}$for the equation applied in the exterior region $\rho>R$ and $\theta(R)=\theta^{-}$ in interior region $\rho<R$. Since the flow is centripetal, the concentration decreases with $\rho$ in 
both domains (Fig. 6), but it should never leave the bistability interval $\theta>\theta_{c}^{+}$or $\theta<\theta_{c}^{-}$ where $\mu_{c}^{-}<f(\theta)<\mu_{c}^{+}$. Applying this restriction in the interior region gives the condition defining the maximum spot radius:

$$
\int_{0}^{R} v(\rho) d \rho=\int_{\theta^{-}}^{\theta_{c}^{-}} \frac{f^{\prime}(\theta)}{\theta} d \theta \equiv \mathcal{N}^{-}(\nu) .
$$

The integral in the left-hand side equals to $-M R \Delta \mathcal{V}^{-}$, where

$$
\mathcal{V}^{-}=\frac{1}{2 \pi} \int_{0}^{1}\left[\frac{1+\rho^{2}}{\rho(1+\rho)} \mathbf{K}\left(\frac{4 \rho}{1+\rho^{2}}\right)-\frac{1+\rho}{\rho} \mathbf{E}\left(\frac{4 \rho}{1+\rho^{2}}\right)\right] d \rho \approx 0.1817
$$

(5.arabicequation)

This yields the upper limit on the spot radius $R \leq R_{m}=\mathcal{N}^{-}(\nu) /\left(M \Delta \mathcal{V}^{-}\right)$.

It might be also impossible to extend the solution to the exterior of the spot beyond a certain finite distance. The upper limit $R_{c}$ is defined implicitly by the relation

$$
\int_{R}^{R_{c}} v(\rho) d \rho=\int_{\theta_{c}^{-}}^{\theta^{+}} \frac{f^{\prime}(\theta)}{\theta} d \theta \equiv \mathcal{N}^{+}(\nu) .
$$

(5.arabicequation)

The critical radius extends to infinity only when the Marangoni number is sufficiently small, so that $M R \Delta \leq R_{c}=\mathcal{N}^{+}(\nu) / V^{+}$, where

$$
\mathcal{V}^{+}=\frac{1}{2 \pi} \int_{1}^{\infty}\left[\frac{1+\rho^{2}}{\rho(1+\rho)} \mathbf{K}\left(\frac{4 \rho}{1+\rho^{2}}\right)-\frac{1+\rho}{\rho} \mathbf{E}\left(\frac{4 \rho}{1+\rho^{2}}\right)\right] d \rho \approx 0.3183
$$

(5.arabicequation)

When the function $f(\theta)$ is symmetric, $\mathcal{N}^{+}<\mathcal{N}^{-}$, and the ratio $\mathcal{N}^{-} / \mathcal{N}^{+}$grows with $\nu$; e.g. for $\nu=16$ we have $\mathcal{N}^{-} \approx 7.636, \mathcal{N}^{+} \approx 1.644$. In this particular case, $M R_{m} \approx 59.44$ and $M R_{c} \approx 7.305$. The largest spot which may exist in this case alone in the infinite plane has the radius $R_{c} \approx 7.305 / M$. The respective concentration profile is drawn in Fig. 6; inside this or smaller drop, the concentration changes only slightly. Beyond this limit, other spots must nucleate at a certain distance, as the chemical potential crosses the bistability limit $\theta_{c}^{+}$. The dependence of $R_{m}$ and $R_{c}$ on $\nu$ is shown in Fig. 7;

When multiple spots exist, the circular symmetry is lost, and analytical computation is no longer possible. Attraction of spots leads to coalescence, and, as the size of a combined spot increases, other spots must nucleate at a certain distance. This leads to chaotic dynamics observed in Ref. [1]. The analytical theory suggests that the typical spot radius should scale as $1 / M$, while a weaker dependence was detected in Ref. [1]. This discrepancy, apparently, stems from the fact that the conditions $M \ll 1, R \gg 1$ have not been observed in the computational study.

\section{Acknowledgments}

The author acknowledges support by the European Union under Grant No. PITN-GA214919. 


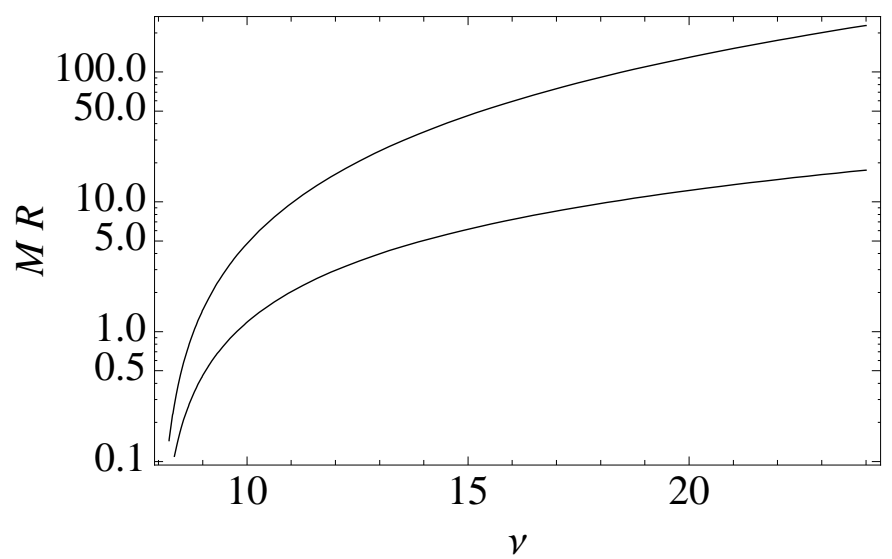

Figure 7: The dependence of the maximum drop radius $R_{m}$ (upper curve) and the maximum radius of a solitary spot $R_{c}$ (lower curve) on the parmeter $\nu$. The radii are normalized by the Marangoni number.

\section{References}

[1] A. A. Golovin and L. M. Pismen. Dynamic phase separation: From coarsening to turbulence via structure formation. Chaos, 14 No. 3, (2004) 845-854.

[2] A. M. Turing. The chemical basis of morphogenesis. Philos. Trans. R. Soc. London B, 237 (1952), 37-72.

[3] R. Kapral and K. Showalter (Eds.). Chemical waves and patterns. Kluwer Academic Publishers, New York, 1995.

[4] L. M. Pismen. Patterns and Interfaces in dissipative dynamics. Springer Verlag, Berlin, 2006.

[5] Z. Dagan and L. M. Pismen. Marangoni waves induced by a multistable chemical reaction on thin liquid films. J. Coll. Interface Sci., 99 (1984), No. 1, 215-225.

[6] L. M. Pismen. Composition and flow patterns due to chemo-Marangoni instability in liquid films. J. Coll. Interface Sci., 102 (1984), No. 1, 237-247.

[7] A. Pereira, P. M. J. Trevelyan, U. Thiele, and S. Kalliadasis. Dynamics of a horizontal thin liquid film in the presence of reactive surfactants, Phys. Fluids, 19 (2007), No. 11, 112102.

[8] L. Rongy and A. De Wit. Solitary Marangoni-driven convective structures in bistable chemical systems. Phys. Rev. E, 77 (2008), No. 4, 046310.

[9] L. M. Pismen. Interaction of reaction-diffusion fronts and Marangoni flow on the interface of deep fluid. Phys. Rev. Lett., 78 (1997), No. 2, 382-385. 
[10] L. M. Pismen and J. Rubinstein. Motion of vortex lines in the Ginzburg-Landau model. Physica (Amsterdam) D, 47 (1991), No. 3, 353-360.

[11] R. L. Pego. Front migration in the nonlinear Cahn-Hilliard equation. Proc. Roy. Soc. Ln A, 422 No. 1863 (1989), 261-278. 